DOSSIER

\title{
Logistique et entreprise virtuelle
}

sous la direction de

Gilles Paché 
INTRODUCTION

PAR GILLES PACHÉ

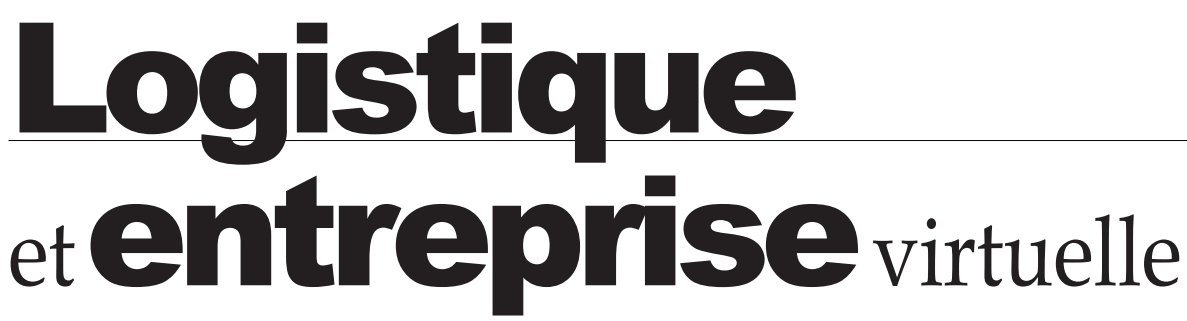

$\mathrm{P}$ our certains observateurs, peu attentifs des évolutions de la logistique depuis une dizaine d'années, il peut apparaître paradoxal d'y associer le thème de l'entreprise virtuelle. En effet, rien de moins « virtuel » que la logistique, du moins lorsque l'on s'intéresse aux énormes moyens matériels et humains impliqués dans son fonctionnement, à l'échelle de pays et, plus encore, à l'échelle de continents. Un ouvrage de vulgarisation publié en France en 2004 à l'initiative de l'association AFILOG, sous le titre En toute logistique (Éditions Jacob-Duvernet, Paris), n'a ainsi de cesse de mettre en scène les réalisations immobilières les plus remarquables des dernières années en matière de plates-formes logistiques. Comme si la puissance de la logistique se mesurait finalement à l'aune d'un gigantisme comptabilisé en centaines de milliers de mètres carrés d'entreposage, sans parler des infrastructures terrestres, portuaires et aéroportuaires permettant de supporter des échanges de produits de plus en plus lointains, mais parfois si proches en termes de délai.

Pourtant, il y a bien une légitimité forte à s'intéresser à l'entreprise virtuelle sous l'angle de la logistique, mais sans doute moins dans une logique de ressources et d'équipements que dans une logique de coordination. Il 
ne fait plus aucun doute aujourd'hui que penser un management logistique efficace, c'est d'abord faire référence à des modes adaptés de pilotage des flux (de produits et d'informations associées) entre des partenaires industriels et commerciaux impliqués collectivement dans un processus de création de valeur. Or, plus que toute autre, l'entreprise dite virtuelle repose sur un management logistique parfaitement organisé, seule manière d'éviter des effets entropiques destructeurs de valeur (médiocre qualité de service au client, augmentation des coûts, etc.). Car finalement, qu'est-ce qu'une entreprise virtuelle? Certes pas une entreprise que l'on ne voit pas! Au contraire, il s'agit d'une forme organisationnelle originale regroupant plusieurs partenaires dont l'objectif est de permettre la mise en œuvre d'effets de synergies ou le développement de capacités/potentiels supplémentaires. Un peu comme une « mémoire virtuelle » ouvre la possibilité à un ordinateur d'être utilisé comme s'il avait plus de mémoire qu'il en a effectivement... Mais la concrétisation de ces capacités/potentiels supplémentaires restera évidemment lettre morte si les interfaces logistiques entre partenaires sont défaillantes.

De nombreux articles ont été consacrés jusqu'à présent aux entreprises virtuelles, encore dénommées, selon les auteurs, entreprises en réseau, entreprises transactionnelles, entreprises modulaires, etc. On pense notamment ici à des travaux conduits en management stratégique, en théorie des organisations, en marketing, en systèmes d'information ou en gestion des ressources humaines. Il est vrai que la dématérialisation des chaînes de valeur soulève de redoutables problèmes dont les chercheurs en sciences de gestion et en analyse industrielle sont loin d'avoir fait le tour. L'objectif du présent dossier n'est pas de fournir une vue générale sur l'entreprise virtuelle, mais de privilégier un angle d'attaque particulier: l'organisation et le management de ses processus logistiques. Le chantier est manifestement immense, mais à peine entamé aujourd'hui. Parmi les interrogations majeures, plusieurs restent ainsi en suspens, et constituent, à n'en point douter, des pistes de recherche de première importance: quels outils d'évaluation de la performance logistique sont les plus pertinents? Comment repérer puis gérer les interfaces logistiques « critiques »? Quelles seront les modalités d'appropriation, par les salariés des différents partenaires, des technologies innovantes indispensables au copilotage des flux ?, etc.

Les auteurs des cinq articles présentés ici ont souhaité adopter des angles d'attaque originaux de la problématique «logistique» dans un contexte de virtualisation. Loin de tout souci d'exhaustivité, leur volonté commune est d'éclairer une facette particulière du phénomène, en mobilisant à chaque fois un cadre conceptuel jugé pertinent et de multiples exemples et illustrations dont la finalité est de mettre en lumière un certain nombre d'enjeux concrets. Il ne s'est donc pas agi pour eux de proposer des « recettes » pour réussir le passage de l'entreprise traditionnelle à l'entreprise virtuelle, mais plutôt d'indiquer aux chercheurs et aux praticiens l'existence de signaux faibles dans la mutation actuelle des formes organisationnelles, tant en termes de freins que d'opportunités de développement. Par-delà les spécialistes de logistique et, plus largement de supply chain management, le dossier s'adresse à 
un lectorat soucieux de comprendre et d'anticiper les impacts de cette mutation.

Dans un premier article, Jacques Colin s'appuie sur une expérience unique d'observateur des stratégies logistiques depuis vingt-cinq ans pour retracer ce que sont, d'après lui, les deux principales phases de déploiement de la logistique: d'abord une gestion des flux de l'entreprise, ensuite une gestion de l'entreprise par les flux. Il en ressort l'idée-clé que le management logistique et stratégique a progressivement élargi son champ d'intervention en passant d'une vision intra-organisationnelle à une vision interorganisationnelle, impliquant de ce fait un nombre croissant de parties prenantes dans la gestion effective par les flux. L'auteur insiste plus particulièrement sur les logiques d'interaction qui structurent la supply chain (interne, puis externe), en faisant le pari que dans un futur proche, les supply chains seront par nature éphémères et fonctionneront de plus en plus systématiquement sur un mode projet.

Dans un deuxième article, Nathalie FabbeCostes creuse entre autres cette dernière idée, en soulignant que les entreprises virtuelles correspondent de plus en plus systématiquement à des sortes de « réseaux organiques » mobilisant des supply chains fondées sur des arrangements de nature temporaire. L'objectif est alors d'activer des acteurs et des ressources pour concrétiser une proposition collective, quitte à voir la chaîne se décomposer une fois le projet réalisé. L'auteur insiste sur les contraintes que sous-tend la capacité à assembler puis désassembler des chaînes très vite opérationnelles, sans que les coûts de changement soient exorbitants. Elle utilise pour cela l'image du «plug and play/unplug », en soulignant finalement que la réussite d'une gestion dynamique des supply chains des entreprises virtuelles reposera sans doute, à l'avenir, sur une parfaite assimilation de compétences de management de réseau.

Dans un troisième article, François Fulconis et Gilles Paché s'intéressent tout particulièrement au cas des prestataires de services logistiques (PSL). Ces derniers apportent de plus en plus aux entreprises virtuelles une compétence de gestion des interfaces participant activement à la mise à disposition des produits aux clients dans les meilleures conditions de coût, de délai et de qualité de service. Les auteurs retracent rapidement le contour des activités prises en charge par les PSL, acteurs des supply chains finalement assez peu connus, et signalent un élargissement significatif de leur offre vers des opérations para-industrielles, comme l'assemblage terminal de composants. Un tel élargissement pourrait peut-être conduire certains PSL dynamiques à devenir progressivement les « pilotes » des entreprises virtuelles de demain.

Dans un quatrième article, Richard Calvi, Éric Blanco et Tetsu Koike défendent la position suivante: la logistique d'une entreprise virtuelle sera d'autant plus efficace et réactive qu'elle se fondera sur un processus de développement privilégiant, dès la phase de conception d'un nouveau produit, des pratiques de coopération où les acteurs de la supply chain du produit concerné se retrouvent au sein d'un « espace commun » de compréhension des problèmes. En s'appuyant sur de nombreux exemples, les auteurs soulignent l'importance de la prise en compte au plus tôt des contraintes logistiques, ce qui doit permettre d'accélérer le processus de mise sur le marché. Seule une 
134 Revue française de gestion

forte implication des différents partenaires constituant la structure de l'entreprise virtuelle peut permettre d'y parvenir, et ainsi participer à la constitution de supply chains « agiles ».

Avec le cinquième et dernier article, Hervé Fenneteau et Gérald Naro nous permettent de « tordre le cou » à certaines idées reçues sur la manière de conduire un partenariat dans les supply chains, en s'appuyant pour cela sur une synthèse critique de la littérature académique. Si la confiance qui s'ins- taure entre les acteurs se présente comme un important catalyseur des partenariats, donnant la possibilité à l'entreprise virtuelle de perdurer et de se développer, elle n'exclut en rien la nécessité d'un contrôle des comportements. Plusieurs exemples de chantiers collaboratifs, tels que la GPA ou le CPFR, illustrent parfaitement selon les auteurs le dualisme confiance/contrôle. Ce dualisme constitue, à n'en point douter, un thème de réflexion majeur dans une perspective décisionnelle. 